\title{
Label-Free Detection of Tobramycin in Serum by Transmission- Localized Surface Plasmon Resonance
}

Giulia Cappi, ${ }^{\dagger}$ Fabio M. Spiga, ${ }^{\dagger}$ Yessica Moncada, ${ }^{\ddagger}$ Anna Ferretti, ${ }^{\dagger}$ Michael Beyeler, ${ }^{\dagger}$ Marco Bianchessi, $^{\ddagger}$ Laurent Decosterd, ${ }^{\S}$ Thierry Buclin, ${ }^{\S}$ and Carlotta Guiducci*, ${ }^{\dagger}$

${ }^{\dagger}$ Institute of Bioengineering, École polytechnique fédérale de Lausanne, Lausanne, CH-1015 Switzerland

${ }^{\ddagger}$ Bio-Lab, STMicroelectronics, Agrate Brianza, Monza and Brianza, 20864, Italy

${ }^{\S}$ Laboratory and Division of Clinical Pharmacology, Lausanne University Hospital (CHUV), Lausanne, CH-1011 Switzerland

\section{Supporting Information}

\begin{abstract}
In order to improve the efficacy and safety of treatments, drug dosage needs to be adjusted to the actual needs of each patient in a truly personalized medicine approach. Key for widespread dosage adjustment is the availability of point-of-care devices able to measure plasma drug concentration in a simple, automated, and costeffective fashion. In the present work, we introduce and test a portable, palm-sized transmission-localized surface plasmon resonance (T-LSPR) setup, comprised of offthe-shelf components and coupled with DNA-based aptamers specific to the antibiotic tobramycin $(467 \mathrm{Da})$. The core of the T-LSPR setup are aptamer-functionalized gold nanoislands (NIs) deposited on a glass slide covered with fluorine-doped tin oxide (FTO), which acts as a biosensor. The gold NIs exhibit localized plasmon resonance in the visible range matching the sensitivity of the complementary metal oxide semiconductor (CMOS) image sensor employed as a light detector. The combination of gold NIs on the FTO substrate, causing NIs size and pattern irregularity, might reduce the overall sensitivity but confers extremely high stability in high-ionic solutions, allowing it to withstand numerous regeneration cycles without sensing losses. With this rather simple T-LSPR setup, we show real-time label-free detection of tobramycin in buffer, measuring concentrations down to $0.5 \mu \mathrm{M}$. We determined an affinity constant of the aptamer-tobramycin pair consistent with the value obtained using a commercial propagating-wave based SPR. Moreover, our label-free system can detect tobramycin in filtered undiluted blood serum, measuring concentrations down to $10 \mu \mathrm{M}$ with a theoretical detection limit of 3.4 $\mu \mathrm{M}$. While the association signal of tobramycin onto the aptamer is masked by the serum injection, the quantification of the captured tobramycin is possible during the dissociation phase and leads to a linear calibration curve for the concentrations over the tested range $(10-80 \mu \mathrm{M})$. The plasmon shift following surface binding is calculated in terms of both plasmon peak location and hue, with the latter allowing faster data elaboration and real-time display of the results. The presented T-LSPR system shows for the first time label-free direct detection and quantification of a small molecule in the complex matrix of filtered undiluted blood serum. Its uncomplicated construction and compact size, together with the remarkable performances, represent a leap forward toward effective point-of-care devices for therapeutic drug concentration monitoring.
\end{abstract}

$\mathrm{T}$ ailoring drug treatments for each patient in a personalized medicine approach holds the promise of dramatically improving therapeutic outcomes and safety. At present, for a vast majority of human medicines, a standard initial dosage is still defined on the basis of population studies and applied uniformly to the patients. However, certain drugs demonstrate remarkably large interindividual variability in their absorption and disposition rates (i.e., their pharmacokinetic characteristics). This leaves a large part of the patients with under- or overexposure in terms of circulating drug concentrations, thus possibly resulting in inefficacy or adverse effects, respectively. In the absence of an early, predictive, easily monitored biomarker, treatment failure or toxicity must become clinically manifested to trigger dosage adaptation. Circulating drug concentration represents a suitable surrogate marker for treatment monitoring and readjustment in such situations. It is available for a limited number of drugs hitherto, and it relies on distant central laboratories equipped with large, costly apparatus. Therefore, there is an important need for miniaturized and affordable systems that could simplify the quantification of drugs in the patient's blood, allowing the blood analysis to be completed at the medical doctor's office. Most of the current drugs are small molecules (m.w. < $1000 \mathrm{Da}$ ) circulating at or below micromolar concentrations. These molecules are chemically stable and often devoid of reactive moieties available for labeling, which makes them difficult to detect and quantify. Their determination in biological fluids through a point-of-care (POC) system thus represents a definite challenge.

Received: January 29, 2015

Accepted: March 26, 2015

Published: March 26, 2015 
Among other techniques, surface plasmon resonance (SPR) has gained importance in the last decades as real time and labelfree surface technique for molecular detection. ${ }^{1-4}$ The SPR phenomenon occurs when light with a particular wavelength is shone at the interface between a thin layer of conductive metal (often gold) and a dielectric, yielding an evanescent plasmon wave that is highly sensitive to the refractive index (RI) in the surroundings of the interface. ${ }^{5,6}$ When the metal surface is functionalized with a ligand, analyte binding causes a local increase of the RI that can be followed and directly measured by a red-shift of the SPR. ${ }^{7}$ Direct detection of small molecules by SPR can be challenging, since their small mass causes only minor RI changes on the surface, which are difficult to detect. Nevertheless, Chang and coauthors recently demonstrated the direct measurement of several small molecules with a commercial propagating SPR system and DNA aptamers, ${ }^{8}$ with detected concentrations varying between a few $\mu \mathrm{M}$ and tens of nM. This shows that, albeit in buffer solution, it is possible to detect small molecules in a label-free setting via propagating SPR.

In order to measure drug concentration in patient samples, however, SPR detection must take place in more complex matrices than buffers. SPR measurement of a cardiac biomarker (brain natriuretic peptide, BNP) in undiluted blood serum was shown in a commercial SPR system using DNA aptamers on the chip surface, and nanoparticles (NP) coated with antibodies $\mathrm{Ab})$ to generate and enhance the signal. ${ }^{9}$ The relatively high molecular weight of $\mathrm{BNP}(>3 \mathrm{kDa})$ and the complex labeling system allow the authors to attain a limit of detection (LOD) in the range of aM. Going toward a portable commercial SPR setup, another work shows the detection of a small molecule (cortisol) in the complex matrix of human saliva. ${ }^{10}$ Once again, in order to increase the signal generated from the small molecule, a competitive assay with a monoclonal antibody $(\mathrm{Ab})$ was used. Interestingly, an in-line filtering system was introduced in order to reduce the matrix complexity, therefore strongly decreasing the amount of large molecules that could reach the SPR surface. Although it is possible to scale it down, the propagating SPR systems described above often require complex optics and scanners difficult to miniaturize.

Another SPR approach, called localized-SPR (LSPR) employs a disconnected pattern of thin metal to generate a nonpropagating plasmon. ${ }^{11-15}$ In the transmission configuration (T-LSPR), the LSPR, excited by the passing-through light, can be readily characterized from the transmitted light, thus greatly simplifying the detection system. ${ }^{16-18}$ Due to its nonpropagating nature, LSPR is also prone to dramatically increase throughput, allowing simultaneous measurements of multiple analytes. ${ }^{19}$ A recent work shows the LSPR detection of a small molecule (ATP) through a simple system that promises high throughput and can be further miniaturized. ${ }^{20}$ However, the detection is only tested in buffer, and it is further complicated by the use of two types of NPs, of which one is modified with the specific aptamer and the other with a complementary short ssDNA. Aćimović and colleagues moved a step forward by measuring several biomarkers in $50 \%$ blood serum via a high throughput $\mathrm{LSPR}^{21}$ in both direct and sandwich assays. Nevertheless, the cancer-specific biomarkers analyzed (alpha-feto-protein and prostate specific antigen) have a molecular mass between 34 and $70 \mathrm{kDa}$ and are therefore not small molecules.

In this paper, we show for the first time direct detection and measurement of a small molecule (tobramycin, molecular weight of $467 \mathrm{Da}$ ) in undiluted blood serum via a simple transmission LSPR system. The T-LSPR setup is based on gold nanoislands (NIs) self-assembled on a glass slide covered with a fluorine-doped tin oxide (FTO) layer. This T-LSPR has a very simple, compact, and cost-effective construction while retaining the capability of an increased throughput by using a complementary metal oxide semiconductor (CMOS) image sensor as a light detector. In parallel to the T-LSPR setup, two algorithms to extract the SPR information were also developed and tested in this work. The T-LSPR system gives excellent results in buffer and, in undiluted blood serum, is almost able to attain the minimal analytical concentration currently required in the clinics.

\section{EXPERIMENTAL SECTION}

Microfluidic Plasmonic Chip for Molecular Analytics. The microfluidic chip is composed of a glass slide coated with gold nanostructures and a microfluidic module which consists of two independent channels. Gold nanoislands (NIs) are formed on fluorine-doped tin oxide (FTO)-coated glass (45 $\mathrm{mm} \times 12 \mathrm{~mm} \times 2 \mathrm{~mm}$ ) by direct thermal evaporation and subsequent thermal annealing. The obtained nanostructures exhibit plasmonic resonance in the visible range $(570 \mathrm{~nm})$ and are extremely stable under flow conditions, due to the fact that gold penetrates into the FTO layer during the thermal annealing step. ${ }^{22}$ A detailed description of the NIs formation process can be found in previous works. ${ }^{23,24}$

Microfluidic channels for the delivery of solutions to the NIs surface are obtained by sticking on the top of the FTO-glass slide a PMMA cover plate: the intermediate stick layer is a made out of double coated tape (3 M High Performance Double Coated Tape 9086) on which two regions of $2 \mathrm{~mm} \times$ $14 \mathrm{~mm} \times 150 \mu \mathrm{m}$ have been removed by laser micromachining, leading to two channels of identical size. Inlets and outlets are $0.8 \mathrm{~mm}$ in diameter such that microfluidics connections are possible from standard syringe needles (Terumo) connected to the syringe pump (Harvard Apparatus 11 Elite Nanomite Programmable Syringe Pump) via flexible silicon tubes (SaniTech STHT-C).

Surface Functionalization and Sample Preparation. The surface of the NIs is modified on different regions of the slide with organic layers immediately after the thermal annealing. The formation of the layers is obtained by spotting $50 \mu \mathrm{L}$ volume solutions and incubating at room temperature for $16 \mathrm{~h}$. The solutions deposited contain either $3 \mu \mathrm{M}$ of the DNA aptamer specific for tobramycin ${ }^{25}$ (SH5'-TCCGTGTATAGGTCGGGTCTCTTGCCAACTGATTCGTTGAAAAGTATAGCCCCGCAGGG-3'; Sigma) in PBS buffer or $1 \mathrm{mM}$ of 6-mercapto-1-hexanol ( $\mathrm{MCH}$, Sigma) in Tris-EDTA 10:1 $\mathrm{mM}$ buffer (TE $1 \times)$ as a control spot. The spots occupy an area of about $20 \mathrm{~mm}^{2}$.

The immobilization of the molecules is based on covalent sulfur-gold bonds. After the incubation, the slides are rinsed with ultrapure water and gently dried with nitrogen. The surface is then passivated with $\mathrm{MCH} 1 \mathrm{mM}$ in TE $1 \times$ for 30 min. Finally, the samples are thoroughly rinsed with ultrapure water and dried by a nitrogen stream. Tobramycin is diluted either in TE $1 \times$ buffer or in FBS (fetal bovine serum, cell culture grade). FBS solutions (with or without tobramycin) were subsequently filtered with an Amicon Ultra Filter (Millipore, Switzerland) with $3 \mathrm{kDa}$ cutoff in order to remove most of the protein content. $\mathrm{NaCl}, \mathrm{PBS}$, and fetal bovine serum 

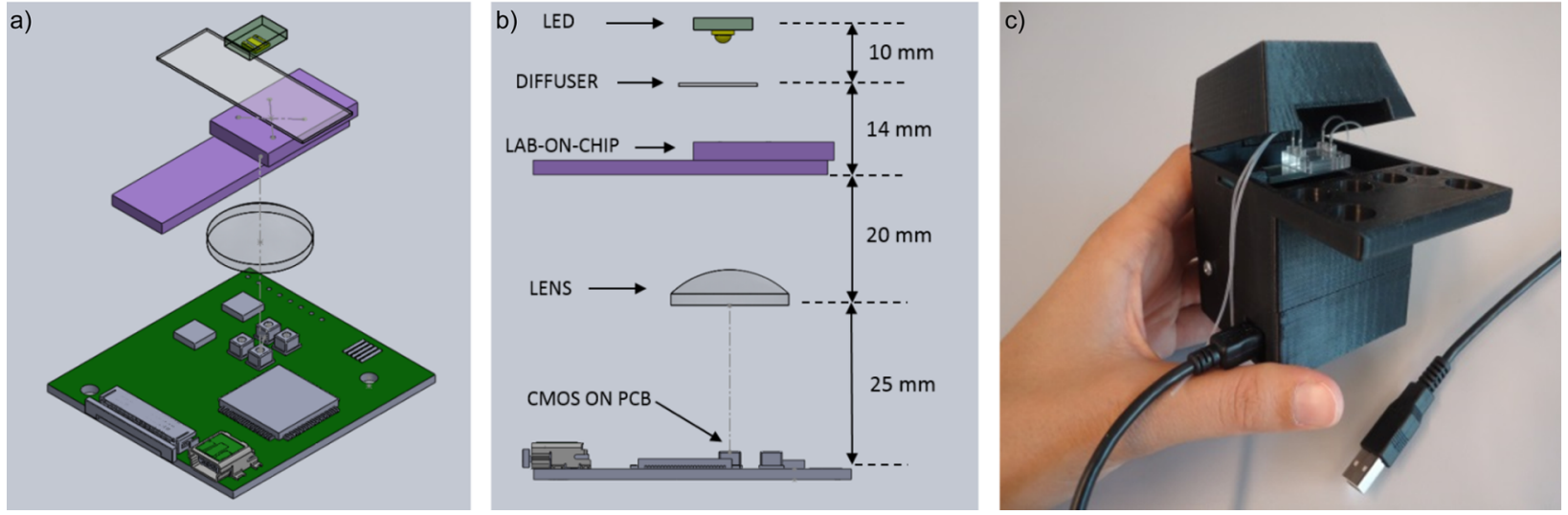

Figure 1. Custom-made T-LSPR setup. (a) Digital rendering of the components of the T-LSPR setup. (b) Side view with relative distances indicated. (c) Picture of the complete setup.

(FBS) were all purchased from Sigma-Aldrich, while TE buffer was purchased from Applichem.

Measurement Setup. The T-LSPR setup employs a nonpolarized white light LED as excitation source (Cree Lamp XP-E supplied by $2.49 \mathrm{~V}$ ) and a CMOS image sensor (ST Microelectronics VW6558) as light detector. The light transmitted through the NI-coated slides contains information on the plasmonic resonance phenomenon occurring at the NIs-solution interface. The light is collected on the CMOS detector by a plano-convex lens (Thorlabs LA1270-A) that reduces the focal distance between the NI-coated slide and the CMOS sensor. Relative distances among the elements of the optical setup are represented in in Figure 1a,b. The use of a CMOS sensor allows one to image a large area of the slide and to distinguish the light absorbed by the different regions pertaining to immobilized aptamer molecules or to control spots bearing $\mathrm{MCH}$.

The CMOS sensor is $3.5 \times 3.03 \times 2.5 \mathrm{~mm}^{3}$ in size. It has an integrated lens with focal length of $1.25 \mathrm{~mm}$ and horizontal field of view (HFOV) of $60^{\circ}$. The resolution is $648 \times 488$ pixels of $2.2 \mu \mathrm{m} \times 2.2 \mu \mathrm{m}$ size each, with red (R), green (G), and blue (B) filters arranged in a Bayer pattern configuration. A diffusor is placed at a $10 \mathrm{~mm}$ distance from the LED to improve light uniformity and to prevent hot spots on the image. All the setup components are placed inside a 3D printed case of $5.5 \mathrm{~cm}$ $\times 6 \mathrm{~cm} \times 8.5 \mathrm{~cm}$ in size which is developed in a dark material to reduce internal reflections (Figure 1c).

Data Analysis. Images of $200 \times 300$ pixels were recorded at 8 frames per second with an analog gain set to 1 ; they were saved in a sequential order in bitmap format and finally were displayed in real-time on the screen. Regions of interest (ROIs) of about 800 pixels were manually selected on the images in order to include the previously spotted areas (Figure 2, red rectangles). RGB intensities were then averaged for each ROI yielding to the corresponding output signal used for further data analysis. First, R, G, and B values for the light transmittance are mediated over the ROI. Successively, the images are analyzed to determine the resonance peak wavelength by means of an algorithm previously developed. ${ }^{23}$ The binding of molecular species on the NIs causes a red-shift in the plasmon peak, while the shape of the resonance itself is only slightly affected.

The characteristic shape of the plasmon resonance is determined with a commercial spectrophotometer (Ocean Optics USB200+) and then used as a reference by the

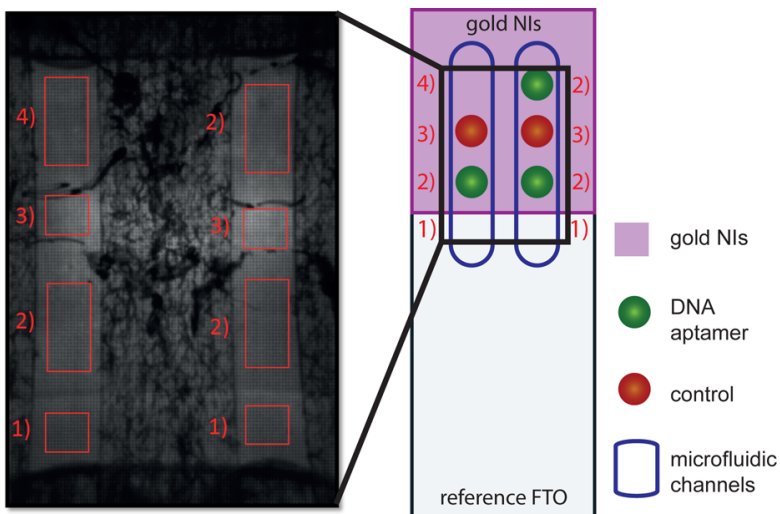

Figure 2. Plasmonic sensor. Schematic representation of a NIs FTO glass slide indicating the spots on the surface and the areas exposed to the microfluidic channels (right). An example of an image acquired by the CMOS camera (left). The ROIs are selected during the data processing. (1) Reference FTO surface. (2) Aptamer spots. (3) MCH control spots. (4) Blank NIs.

algorithm. At each iteration, the reference curve is shifted in wavelength and transmittance until the best fit with the set of three measured points is reached and the peak location is estimated. The algorithm also takes into account the spectral characteristics of the white LED and the RGB filters used in the optical setup (Figure 3). The detailed description of the algorithm can be found elsewhere. ${ }^{23}$

In this work, we exploited an alternative approach to represent the plasmonic resonance in the color space. In the HSV (hue, saturation, value) representation of colors, the hue $(H)$ value corresponds to the perceived color tone and thus indirectly to the physical wavelength; therefore, it is a suitable parameter to evaluate the spectral variations of the plasmon resonance peak in the spectrum. In fact, it solely depends on the measured combination of red, green, and blue pixel values, and it is independent from the overall luminous intensity.

Raw data are first converted from the Bayer pattern to RGB values and then converted into the HSV color space. For each selected ROI, the hue of the average of the selected pixels is calculated and then low-pass filtered (filter order of 126, cutoff frequency of $0.00438 \mathrm{~Hz}$ ).

$$
\begin{aligned}
& \operatorname{MAX}=\operatorname{MAX}(R, G, B) \\
& \min =\min (R, G, B)
\end{aligned}
$$




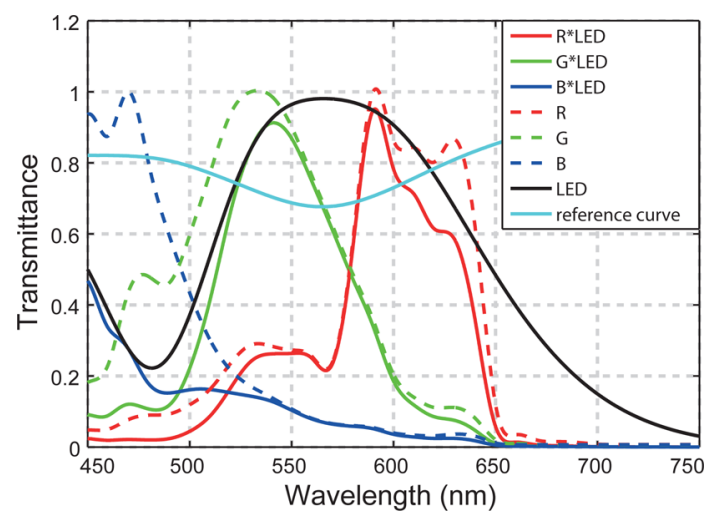

Figure 3. Optical characterization of the plasmon, light source, and light detector employed. Gold NIs spectrum (light blue line) measured by a UV-vis-NIR spectrophotometer Jasco V-570 in transmittance mode with a scan speed of $200 \mathrm{~nm} / \mathrm{min}$ and $1 \mathrm{~nm}$ bandwidth. The solid black line represents the white LED emission. The dashed blue, green, and red lines represent the RGB filters of the CMOS image sensor normalized by their respective maximum values, and the continuous blue, green, and red lines are the same curves convoluted with the LED spectrum.

$$
\begin{aligned}
& H^{\prime}= \begin{cases}\frac{G-B}{\mathrm{MAX}-\min } & \text { if } \mathrm{MAX}=R \\
\frac{B-R}{\mathrm{MAX}-\min }+2 & \text { if } \mathrm{MAX}=G \\
\frac{R-G}{\mathrm{MAX}-\min }+4 & \text { if } \mathrm{MAX}=B\end{cases} \\
& H=\frac{H^{\prime}}{6} \quad \text { if } H<0
\end{aligned}
$$

The variation of the hue was then used to display the plasmonic changes over time.

Stability of the NIs Layer in Aqueous Solution. Stability of the gold NIs was tested by measuring the NIs FTO-glass prior and after $24 \mathrm{~h}$ immersion in TE $1 \times$ buffer. Extinction spectra measured with a commercial spectrophotometer (Ocean Optics 2000+) show no appreciable shift. In order to test the NIs adhesion in the presence of a flow of solution, the microfluidic module was mounted on the NIs FTO-glass slide and TE buffer is flushed for $10 \mathrm{~min}$ at $5 \mu \mathrm{L} / \mathrm{min}$. The negligible change of the peak location proves the stability of the gold NIs under flow.

Real-Time Monitoring of Surface Binding. Microfluidics was built on top of the NIs FTO-glass slide such that two independent channels are covering the areas spotted with the DNA aptamer, the MCH reference spots, a bare gold NIs area, and a blank FTO area (Figure 2). Surface conditioning, consisting of $1 \mathrm{M} \mathrm{NaCl}$ solution followed by $\mathrm{TE} 1 \times$, is performed prior the experiments. Tobramycin in different concentrations is delivered to the sensitive areas and is followed by $\mathrm{TE}$ buffer to observe the dissociation kinetics. $\mathrm{NaCl}(1 \mathrm{M})$ is used as a regeneration solution to remove the remaining tobramycin on the surface and to leave the DNA aptamers on the surface ready for the next cycle.

\section{RESULTS AND DISCUSSION}

Small Molecule Analysis with a Custom-Made LSPR System and DNA Aptamers. DNA aptamers have been widely used for small molecule detection, also in combination with SPR-based systems. ${ }^{8,9}$ We have previously developed a DNA aptamer that can successfully detect the antibiotics tobramycin (m.w. $467 \mathrm{Da}$ ) on propagating SPR (Biacore X100, $\mathrm{GE}$ ), in a label-free assay, down to $50 \mathrm{nM}$ in buffer solution. ${ }^{25}$ We therefore deployed the same aptamer in order to observe the signal generated by the small molecule tobramycin on a nonconnected pattern of plasmonic gold NIs in a transmission localized SPR configuration.

The DNA aptamers were immobilized on the gold NIs via thiol-gold covalent binding, similarly to the passivating selfassembled monolayer of 6-mercapto-1-hexanol. During the injection of solutions at different tobramycin concentrations, molecular binding to the aptamer is detected by monitoring the RI change in the proximity of the NIs, which causes a shift of the plasmon resonance wavelength. Surface drug binding induces an increase of the local RI resulting in a redshift of the plasmon peak. ${ }^{7,11-13,26}$ The real-time shift of the plasmon peak is extracted by a custom developed algorithm (see the Experimental Section), and it is compared with the corresponding change in hue, which has already been used to detect plasmonic changes related to bulk RI measurements ${ }^{27}$ (Figure 4). Despite the small molecular weight of tobramycin, a binding/dissociation curve is clearly detectable, while the control spot $(\mathrm{MCH})$ shows no apparent binding (Figure 4, bottom). The noise on the peak $(0.0715 \mathrm{~nm})$ and on the hue (0.0001) baselines allows one to distinguish the association and dissociation binding interactions. The two approaches for plasmon shift quantification show high consistency, as they exhibit a linear correlation independent from the matrix where the binding event takes place (TE buffer and serum tested, Supporting Information). The difference in the ripples between the hue and peak signals visible in Figure 4 is due to dissimilarities in data treatment (e.g., noise filtering) amid the two approaches, and it is not related to differences in the signal information content. Hue calculation only requires a quick conversion of the RGB values, thus reducing the complexity of the data elaboration and allowing one to plot the results in realtime during the experiment. In addition, the hue is independent from the light intensity; therefore, it is insensitive to the light distribution over the CMOS image sensor. In this work, we show for the first time that hue can be reliably used to measure and display real-time surface binding of small molecules in SPR.

Real-Time Sensing of Tobramycin in TE Buffer. In order to confirm the ability of the T-LSPR setup to quantify tobramycin binding on the DNA aptamers, we sequentially injected tobramycin on the sensing array at different concentrations $(0.5,1,2,5$, and $10 \mu \mathrm{M})$, followed by dissociation (TE buffer) and regeneration solution (1 M $\mathrm{NaCl}$, Figure 5). The hue variation of the DNA aptamerfunctionalized NIs (blue line) and of the control-functionalized NIs ( $\mathrm{MCH}$, green line) was then followed in real-time. A signal proportional to the amount of bound tobramycin is clearly visible in the aptamer-functionalized spots, while the control spot only shows a slight variation of signal probably due to some nonspecific binding and bulk effect. $\mathrm{NaCl}$ brings back the baseline to the value before tobramycin injection, thus efficiently removing the tobramycin bound to the surface and 

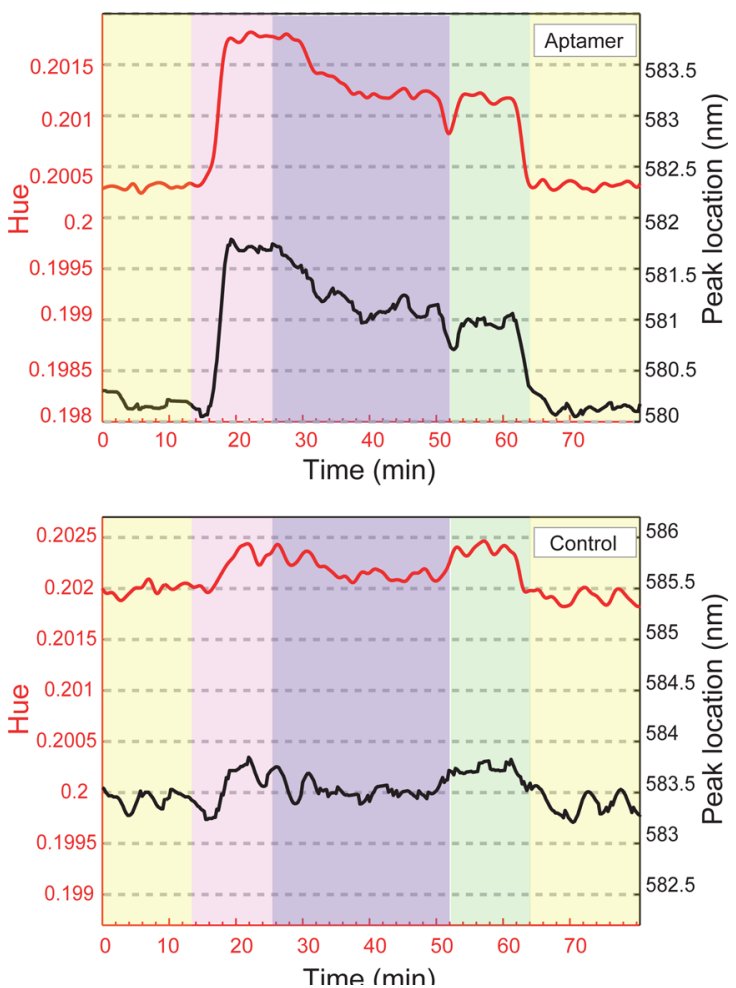

Figure 4. Surface detection of small molecule binding on DNA aptamers. $70 \mu \mathrm{L}$ of tobramycin $(10 \mu \mathrm{M})$ in TE $1 \times$ is delivered to the DNA aptamers (top figure) and to a control $\mathrm{MCH}$ (bottom figure) on the surface at $5 \mu \mathrm{L} / \mathrm{min}$. Peak location (black line) and hue (red line) are extracted from the same RGB data collected by the CMOS image sensor. The pink and purple areas represent the association and the dissociation phase, respectively. The green area represents the regeneration step performed by injection of $\mathrm{NaCl}$ solution. The control spot does not show any specific response to the injection of tobramycin.

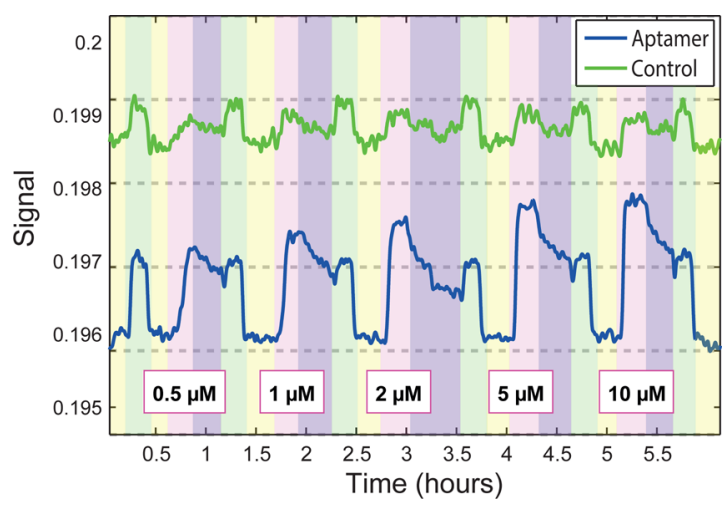

Figure 5. Real-time association and dissociation of tobramycin recognized by the selected DNA aptamer (blue line) attached to the surface. $70 \mu \mathrm{L}$ of tobramycin in concentrations of $0.5,1,2,5$, and 10 $\mu \mathrm{M}$ in TE $1 \times$ are delivered to the surface at $5 \mu \mathrm{L} / \mathrm{min}$. The green line represents the control measurement performed with a layer of $\mathrm{MCH}$ on the NIs, which allows one to cancel out the signal of the bulk effect and to verify the occurrence of nonspecific binding. The pink and purple areas indicate the association and dissociation phases, respectively. The green areas correspond to the injection of $\mathrm{NaCl}$ as a regeneration step.

leaving the DNA aptamers available for capturing drug molecules during the forthcoming cycle.
The effective surface regeneration allows one to reuse the same surface without requiring the functionalization of a new NIs FTO-coated glass slide. The association and dissociation signals for the different concentrations of tobramycin tested increase with the concentration (Figure 6). Injections of 0.5

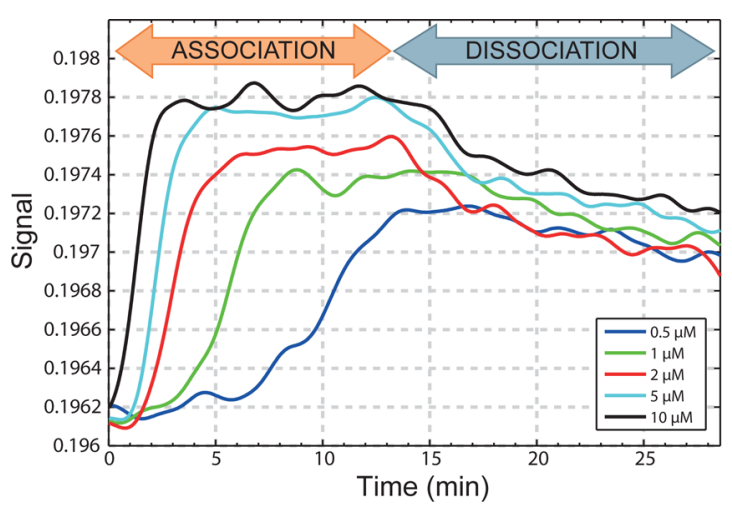

Figure 6. Kinetics association and dissociation of tobramycin for the different concentrations tested.

$\mu \mathrm{M}$ of tobramycin show a slow association rate which is evidently affected by the limiting mass transport phenomenon, while at higher tobramycin concentrations, the association rates become faster. Plotting the association signal values at steadystate with respect to the concentration of tobramycin injected (Figure 7) allows us to fit the points using the Langmuir

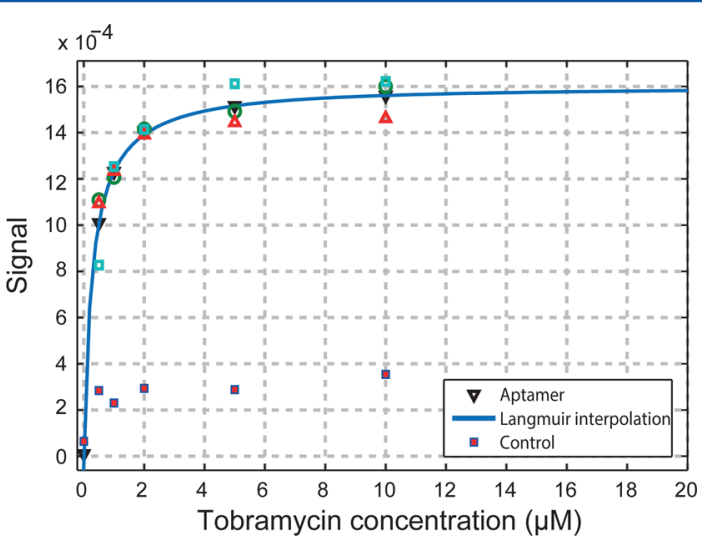

Figure 7. Hue variation for different concentrations of tobramycin averaged over 3 independent DNA aptamer spots on the same NIs FTO glass slide (empty green circles, blue squares, and red triangles) and for the $\mathrm{MCH}$ control spot (red squares).

equation and, hence, to calculate the equilibrium dissociation constant $\left(K_{\mathrm{D}}\right) . K_{\mathrm{D}}$ is indeed the concentration of tobramycin needed to achieve a half-maximum binding and in our case corresponds to $0.26 \mu \mathrm{M}$. The same analysis performed with a commercial SPR gives a $K_{\mathrm{D}}$ of $0.23 \mu \mathrm{M}$ for the same aptamer. ${ }^{25}$ The high similarity of the $K_{\mathrm{D}}$ obtained with our T-LSPR and the commercial SPR indicates that the DNA aptamers are comparably functionalized on the surface, while retaining their ability to recognize tobramycin. Moreover, this suggests that the information from the T-LSPR system is analogous to that of the commercial SPR for the tested concentrations.

Our T-LSPR system can clearly detect tobramycin at least down to the concentration of $0.5 \mu \mathrm{M}$ (minimum tested concentration), while with the same aptamer on the 


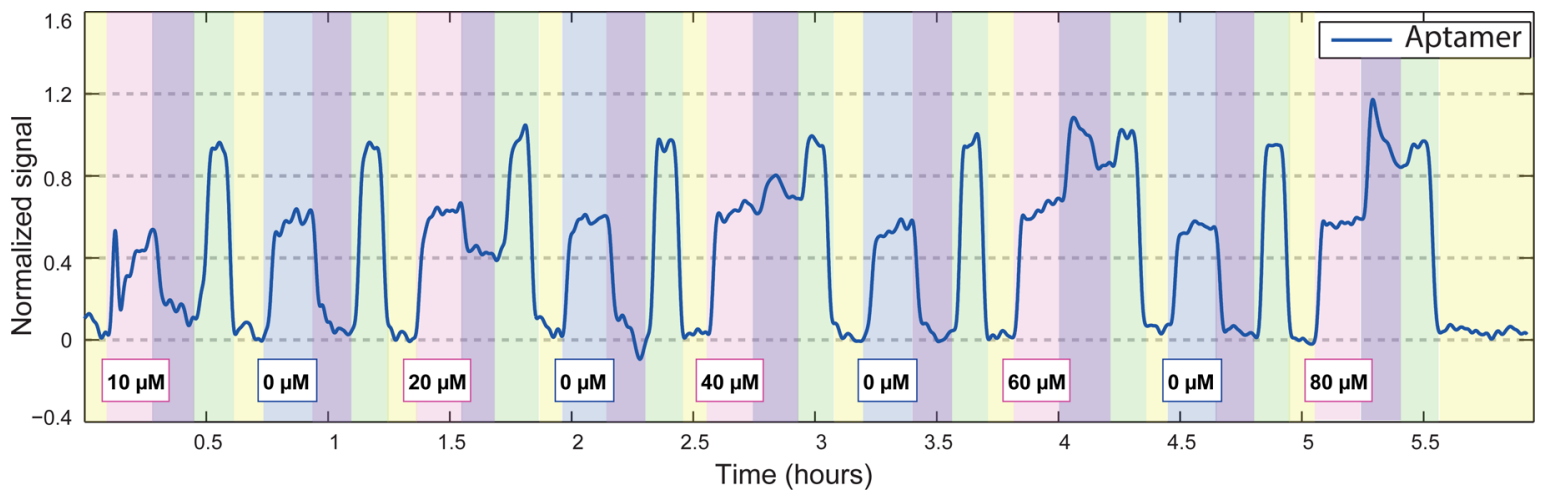

Figure 8. Real-time association and dissociation of tobramycin recognized by the selected DNA aptamer attached to the surface. $70 \mu \mathrm{L}$ of tobramycin with the concentrations of $10,20,40,60$, and $80 \mu \mathrm{M}$ in undiluted serum, alternated with control injections of serum without tobramycin, are delivered to the surface at $10 \mu \mathrm{L} / \mathrm{min}$. The pink and purple areas indicate the association and dissociation phases, respectively. The green areas correspond to the injection of $\mathrm{NaCl}$ as a regeneration step.

commercial propagating SPR, we could detect at least down to $50 \mathrm{nM}$. Chang et al. showed direct assay quantification of several small molecules (m.w. between 75.1 and 690.4 Da) via DNA aptamers in buffer with a commercial propagating SPR system. ${ }^{8}$ Although the sensitivity obviously also depends on the binding strength between ligand and analyte, for molecules of comparable size to tobramycin, they report a similar limit of detection of $0.82 \mu \mathrm{M}$ (ATP, m.w. $506 \mathrm{Da}$ ) and $40 \mathrm{nM}$ (flavin mononucleotide, m.w. $456 \mathrm{Da})$. Xie et al. also tested the detection of ATP reporting a lower limit of detection of 0.01 $\mu \mathrm{M}$ in buffer. $^{20}$ Nevertheless, the label-based approach used takes advantage of gold nanospheres conjugated both to the small molecule and to its DNA aptamer in order to enhance the LSPR signal.

Overall, the custom T-LSPR presented shows data that are comparable to the commercial propagating SPR in terms of the measurement of binding affinity. The apparent sensitivity is, at worst, 1 order of magnitude lower than the commercial SPR, although it remains in line with the sensitivity shown by others for similarly small molecules in label-free assays.

Real-Time Sensing of Tobramycin in Undiluted Serum. A POC system to analyze patient blood with fast and precise response needs to be able to measure tobramycin by binding with the DNA aptamer in the presence of a complex matrix. Therefore, we tested our T-LSPR set up for tobramycin detection in undiluted fetal bovine serum (FBS) as a surrogate for human serum. Because our system is label-free and, thus, prone to be sensitive to nonspecific binding, FBS was filtered with a $3 \mathrm{kDa}$ cutoff membrane in order to remove most of the proteins in the serum (see Experimental Section). ${ }^{10}$

Tobramycin concentrations within the clinical analytical range $(10,20,40,60$, and $80 \mu \mathrm{M}$; clinical range of $0.5-80 \mu \mathrm{M})$ were then spiked in FBS, filtered, and injected at $10 \mu \mathrm{L} / \mathrm{min}$ over the sensing areas in order to be measured with the $\mathrm{T}$ LSPR measurement system (Figure 8). The data were normalized for baseline drift and response variations by the signal corresponding to the regeneration injection $(\mathrm{NaCl} 1 \mathrm{M}$, entirely bulk effect). In order to control for nonspecific binding of molecules, as well as to evaluate serum bulk effect, tobramycin-spiked FBS injections were alternated with injections of pure FBS. The control injections show a pronounced bulk effect because of the higher RI of serum with respect to TE buffer (Figure 8). The signal of the serum samples is constant over the multiple injections, and moreover, it is not affected by the presence of tobramycin (blue and pink areas in Figure 8). Nevertheless, the tobramycin captured by the DNA aptamer is visible and measurable from the start of the dissociation phase (purple areas), when only TE buffer is injected in the channels. The tobramycin-specific signal in the early dissociation phase exhibits a sharp increase to a value related to the amount of tobramycin injected. The control spot (data not shown) and the injections of serum without tobramycin (Figure 8) show no binding signal in that phase.

The reason why serum prevents the observation of the binding of tobramycin to the DNA aptamer during the association is still unclear. One possibility is that the serum could partially absorb the light at the wavelength required for plasmon excitation, hence partially reducing the plasmon efficiency. However, the FBS absorbance spectrum, measured with a commercial spectrophotometer (Nanodrop 2000c Thermo Scientific), only shows peaks at 200 and $300 \mathrm{~nm}$ (data not shown), which are out of the range for the NIs plasmon. On the other hand, filtered blood serum still contains salts, peptides, small proteins, hormones, and further ions. As a result, blood serum has a high load of charges, which has been shown to be sufficient to modify the surface charge of particular molecules $^{28}$ and therefore might reduce the tobramycininduced signal. Additionally, in the context of the metalhalides complex formation, it was shown that the interaction between an electrolyte and a plasmonic metal surface can affect the plasmonic properties of the system. ${ }^{29}$ Therefore, an interesting hypothesis that requires further investigation is that the electrolytes present in the serum might be damping the plasmon, therefore confining the plasmonic electric field to the close proximity of the gold NIs surface. This would reduce the plasmon penetration depth into the microfluidic channel, thus restricting the sensing volume below the height of the tobramycin binding site on the aptamer.

Averaging the dissociation signal of tobramycin on the DNA aptamer spots (Figure 9) shows a linear trend with a sensitivity of 0.01 (hue variation per $\mu \mathrm{M}$ ). The average variability of the two experiments over the different concentrations has been duplicated, summed to the average signal at $0 \mu \mathrm{M}$, and used to estimate the minimum resolvable concentration of tobramycin in serum, which corresponds to $3.4 \mu \mathrm{M}$.

To date, there are no directly comparable works on label-free SPR detection of small molecules. An existing attempt was based on nanoparticle-SPR enhancement, combined with a sandwich assay on a conventional propagating SPR system. ${ }^{9}$ The detection of brain natriuretic peptide (BNP, $3.46 \mathrm{kDa}$ ) in 


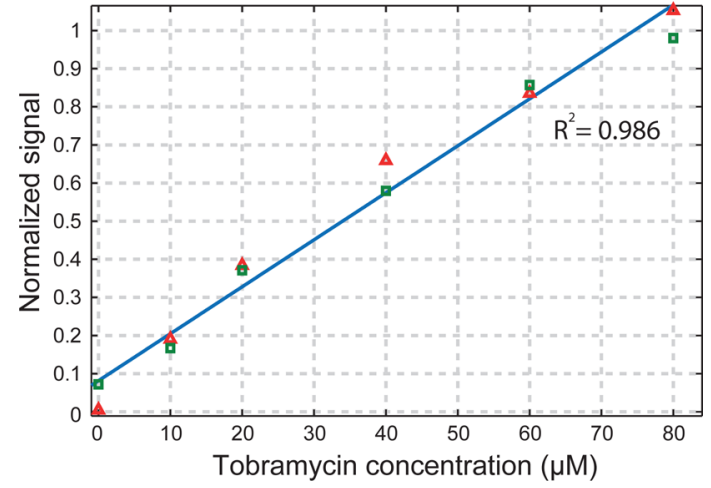

Figure 9. Linear response of the T-LSPR setup to undiluted serum samples spiked with tobramycin. Hue variation for different concentrations of tobramycin averaged over 2 independent DNA aptamer spots on the same NIs FTO glass slide normalized over $\mathrm{NaCl}$ injections.

this work was reported down to $10 \mathrm{aM}$ in serum. Nevertheless, the LOD of BNP is extremely sensitive to the concentration of the nanoparticle-labeled antibody employed for the sandwich assay, making this approach heavily dependent on feedback adjustment. In another work, LSPR direct detection of IgG (m.w. $150 \mathrm{kDa}$ ) in $50 \%$ serum showed a LOD of $0.4 \mathrm{nM}^{21}$ However, $30 \mathrm{~min}$ of measurement is necessary to obtain a detectable signal, due to the competition of the analyte molecules with the abundant serum constituents. With the same setup, slightly smaller analytes, such as alpha-feto-protein (m.w. $70 \mathrm{kDa}$ ) and prostate specific antigen (m.w. $34 \mathrm{kDa}$ ), were detected by means of a sandwich assay approach. Although low LODs were attained (7.1 and $29.4 \mathrm{pM}$, respectively), the employment of a sandwich assay might not be suitable for the detection of small molecules.

The presented T-LSPR system is able to quantify tobramycin with a label-free direct assay in undiluted blood serum. The measured concentrations of tobramycin in patients' blood range between 0.5 and $80 \mu \mathrm{M},{ }^{30}$ and the minimum is less than 1 order of magnitude below the theoretical LOD reported in this work. Interestingly, the DNA aptamer used in this work showed excellent selectivity in propagating SPR toward molecules belonging to the class of coadministered antibiotics (carbenicillin, penicillin-like) and good selectivity against a similar aminoglycoside antibiotic (kanamycin), ${ }^{25}$ therefore confirming its potential in clinical analysis.

The aptamer-modified NIs FTO surface was able to withstand more than $20 \mathrm{~h}$ of repeated binding and regeneration cycles. The remarkable stability of both the aptamer-NIs bonds and the NIs patterns on the FTO well matches a critical requirement for molecular sensing. ${ }^{31,32} \mathrm{We}$ think that this property arises from the porosity of the FTO layer and the subsequent penetration of the NIs in it, as previously suggested. $^{22-24}$ The capability of our T-LSPR sensor to resolve smaller RI changes is limited by the irregularity of the sensor surface which broadens the plasmon and also is due to the FTO granular structure. Nevertheless, attaining more regular shape and patterns of the NIs would allow one to improve the sensitivity. ${ }^{33-37}$

\section{CONCLUSIONS}

With the aim of improving personalized medicine through tailored drug dosage, by allowing small drug quantification in patient blood at the point-of-care, we present in this work a portable T-LSPR setup. Our system is made from off-the-shelf components with dimensions down to a palm size. We fabricated NIs FTO-coated glass slides functionalized with DNA aptamers showing very high stability in solvents and in high ionic-strength solutions. Importantly, these allowed us to perform multiple regeneration cycles without critical degradation of the signal and to determine kinetic parameters that well matched those obtained with a commercial propagating SPR system.

We challenged our device in a label-free configuration by quantifying tobramycin, a low-molecular weight drug that requires personalized dosage. Determination of tobramycin concentrations in TE $1 \times$ buffer down to $0.5 \mu \mathrm{M}$ was attained. Tobramycin detection was also performed in filtered undiluted blood serum, showing an estimated LOD of $3.4 \mu \mathrm{M}$ and a linear concentration dependence of the signal.

This is the first work reporting on a portable T-LSPR system measuring the concentration of small molecules in filtered undiluted serum with a direct assay configuration. At present, the demonstrated detection limit differs from the clinical range by less than 1 order of magnitude. It is therefore not too optimistic to envision that our portable T-LSPR system will be capable of measuring throughout the clinically relevant dynamic range.

\section{ASSOCIATED CONTENT}

\section{Supporting Information}

Supporting figures reporting the relationship between peak wavelength and hue. This material is available free of charge via the Internet at http://pubs.acs.org.

\section{AUTHOR INFORMATION}

\section{Corresponding Author}

*Tel: +41 2169 37813. Fax: +41 2169 31110. E-mail: carlotta. guiducci@epfl.ch.

\section{Author Contributions}

All authors have given approval to the final version of the manuscript.

Notes

The authors declare no competing financial interest.

\section{ACKNOWLEDGMENTS}

This work, scientifically evaluated by the SNSF, has been funded by Nano-Tera.ch (project 128852 "ISyPeM"), an initiative financed by the Swiss Confederation, and it has been funded by the Swiss Commission for Technology and Innovation (CTI) (project 14860.1 PFLS-LS).

\section{REFERENCES}

(1) Rich, R. L.; Myszka, D. G. J. Mol. Recognit. 2010, 23, 1.

(2) Homola, J.; Vaisocherová, H.; Dostálek, J.; Piliarik, M. Methods 2005, 37, 26.

(3) Hoa, X. D.; Kirk, A. G.; Tabrizian, M. Biosens. Bioelectron. 2007, 23, 151 .

(4) Hutter, E.; Fendler, J. H. Adv. Mater. 2004, 16, 1685.

(5) Abbas, A.; Linman, M. J.; Cheng, Q. Biosens. Bioelectron. 2011, 26, 1815.

(6) Otte, M. A.; Sepúlveda, B.; Ni, W.; Juste, J. P.; Liz-Marzán, L. M.; Lechuga, L. M. ACS Nano 2009, 4, 349.

(7) Homola, J. Anal. Bioanal. Chem. 2003, 377, 528.

(8) Chang, A. L.; McKeague, M.; Liang, J. C.; Smolke, C. D. Anal. Chem. 2014, 86, 3273-3278. 
(9) Jang, H. R.; Wark, A. W.; Baek, S. H.; Chung, B. H.; Lee, H. J. Anal. Chem. 2013, 86, 814.

(10) Stevens, R. C.; Soelberg, S. D.; Near, S.; Furlong, C. E. Anal. Chem. 2008, 80, 6747.

(11) Miller, M. M.; Lazarides, A. A. J. Phys. Chem. B 2005, 109, 21556.

(12) Szunerits, S.; Praig, V. G.; Manesse, M.; Boukherroub, R. Nanotechnology 2008, 19, 195712.

(13) Malinsky, M. D.; Kelly, K. L.; Schatz, G. C.; Van Duyne, R. P. J. Am. Chem. Soc. 2001, 123, 1471.

(14) Haes, A. J.; Van Duyne, R. P. J. Am. Chem. Soc. 2002, 124, 10596.

(15) Kedem, O.; Tesler, A. B.; Vaskevich, A.; Rubinstein, I. ACS Nano 2011, 5, 748.

(16) Huang, C.; Bonroy, K.; Reekmans, G.; Laureyn, W.; Verhaegen, K.; De Vlaminck, I.; Lagae, L.; Borghs, G. Biomed. Microdevices 2009, 11, 893.

(17) Ruach-Nir, I.; Bendikov, T. A.; Doron-Mor, I.; Barkay, Z.; Vaskevich, A.; Rubinstein, I. J. Am. Chem. Soc. 2006, 129, 84.

(18) Malic, L.; Morton, K.; Clime, L.; Veres, T. Lab Chip 2013, 13, 798-810.

(19) Lee, S. H.; Lindquist, N. C.; Wittenberg, N. J.; Jordan, L. R.; Oh, S.-H. Lab Chip 2012, 12, 3882.

(20) Xie, L.; Yan, X.; Du, Y. Biosens. Bioelectron. 2014, 53, 58.

(21) Aćimović, S. S.; Ortega, M. A.; Sanz, V.; Berthelot, J.; GarciaCordero, J. L.; Renger, J.; Maerkl, S. J.; Kreuzer, M. P.; Quidant, R. Nano Lett. 2014, 14, 2636.

(22) Montmeat, P.; Marchand, J.-C.; Lalauze, R.; Viricelle, J.-P.; Tournier, G.; Pijolat, C. Sens. Actuators, B 2003, 95, 83.

(23) Cappi, G.; Accastelli, E.; Cantale, V.; Rampi, M. A.; Benini, L.; Guiducci, C. Sens. Actuators, B 2013, 176, 225.

(24) Cantale, V.; Simeone, F. C.; Gambari, R.; Rampi, M. A. Sens. Actuators, B 2011, 152, 206.

(25) Spiga, F. M.; Maietta, P.; Guiducci, C. ACS Comb. Sci. 2015; DOI: 10.1021 /acscombsci.5b00023.

(26) Sherry, L. J.; Jin, R.; Mirkin, C. A.; Schatz, G. C.; Van Duyne, R. P. Nano Lett. 2006, 6, 2060.

(27) Ho, H. P.; Wong, C. L.; Chan, K. S.; Wu, S. Y.; Lin, C. Appl. Opt. 2006, 45, 5819.

(28) Zelphati, O.; Uyechi, L. S.; Barron, L. G.; Szoka, F. C., Jr. Biochim. Biophys. Acta, Lipids Lipid Metab. 1998, 1390, 119.

(29) Dahlin, A. B.; Zahn, R.; Voros, J. Nanoscale 2012, 4, 2339.

(30) Hennig, S.; Standing, J.; Staatz, C.; Thomson, A. Clin. Pharmacokinet. 2013, 52, 289.

(31) Huang, C.; Ye, J.; Wang, S.; Stakenborg, T.; Lagae, L. Appl. Phys. Lett. 2012, 100; DOI: 10.1063/1.4707382.

(32) Barik, A.; Otto, L. M.; Yoo, D.; Jose, J.; Johnson, T. W.; Oh, S.H. Nano Lett. 2014, 14, 2006.

(33) Willets, K. A.; Van Duyne, R. P. Annu. Rev. Phys. Chem. 2007, $58,267$.

(34) Haynes, C. L.; Van Duyne, R. P. J. Phys. Chem. B 2001, 105, 5599.

(35) Kelly, K. L.; Coronado, E.; Zhao, L. L.; Schatz, G. C. J. Phys. Chem. B 2002, 107, 668.

(36) Hong, Y.; Huh, Y.-M.; Yoon, D. S.; Yang, J. J. Nanomater. 2012, 2012, 13.

(37) Anker, J. N.; Hall, W. P.; Lyandres, O.; Shah, N. C.; Zhao, J.; Van Duyne, R. P. Nat. Mater. 2008, 7, 442. 